\title{
Susceptibilidad clonal del álamo (Populus sp.) al ataque de Platypus mutatus en Buenos Aires, Argentina
}

\author{
Cloning susceptibility of Poplar (Populus sp.) when attacked \\ by Platypus mutatus in Buenos Aires, Argentina
}

\author{
Jorge Luis Marquina ${ }^{1 *}$, Raúl Marlats ${ }^{1}$, Marcela Núñez Cresto ${ }^{1}$ \\ *Autor de correspondencia: ${ }^{1}$ Universidad Nacional de la Plata, Facultad de Ciencias Agrarias y Forestales, Diagonal 113 \\ y 61, La Plata, Argentina, código postal 1900, Tel.: 0221-424-4862, jmarqui@ ceres.agro.unlp.edu.ar
}

\begin{abstract}
SUMMARY
The objective of the present work was to characterize the incidence of Platypus mutatus on clones of poplars from plantations located at Palantelén, Alberti, Buenos Aires, Argentina ( $35^{\circ} 40^{\prime} \mathrm{S} ; 60^{\circ} 15^{\prime} \mathrm{W}$ ). 10-year-old plantations of Populus deltoides cvs. Catfish 2, Stoneville 62, Stoneville 66, Stoneville 71 and Populus x euroamericana cvs. I-214 and Conti 12 were studied. The number of galleries by trunk, breaks caused by the insect and survival of broken trees were analyzed. Classifications of clones depending on the pest varied according to the analyzed perturbation. Conti 12 and Stoneville 66 were the most attacked clones measured by presence of galleries. I-214, Stoneville 71 and Stoneville 62 were the least attacked clones. Catfish 2 and Stoneville 66 were more easily broken; followed by Conti 12. I-214 did not have any broken plant. Conti 12 was the clone presenting the least survival of broken plants. Broken plants of Stoneville 71 did not die. Stoneville 62, Stoneville 66, and Catfish 2, presented high survival of broken trees.
\end{abstract}

Key words: Populus sp., cloning susceptibility, damages, Platypus mutatus, Coleopteran, Platypodidae.

\section{RESUMEN}

El objetivo del trabajo fue caracterizar la incidencia de Platypus mutatus en varios clones de Populus de uso comercial, en plantaciones ubicadas en Palantelén, Alberti, Buenos Aires, Argentina (35 40' S, 60 $15^{\circ} \mathrm{O}$ ). Sobre rodales de 10 años de edad de Populus deltoides cvs. Catfish 2, Stoneville 62, Stoneville 66, Stoneville 71, y Populus x euroamericana cvs. I-214 y Conti 12 , se comparó el número medio de galerías por ejemplar, y se evaluaron quebraduras ocasionadas por la plaga y supervivencia de ejemplares quebrados. El ordenamiento de clones resultó variable según la perturbación analizada. Conti 12 y Stoneville 66 fueron los clones más afectados medidos por la presencia de galerías; I-214, Stoneville 71 y Stoneville 62, fueron los menos afectados. Catfish 2 y Stoneville 66, seguidos por Conti, se presentaron como más propensos al quiebre; I-214 no presentó quebraduras. Conti 12 fue el de menor supervivencia; Stoneville 71 no registró muertes, a pesar de las quebraduras; Stoneville 62, Stoneville 66, y Catfish 2, presentaron alta supervivencia de ejemplares quebrados.

Palabras clave: Populus sp., susceptibilidad clonal, daños, Platypus mutatus, Coleoptera, Platypodidae.

\section{INTRODUCCIÓN}

El género Populus (álamos) posee un papel destacado dentro de la producción forestal desarrollada en Argentina. Según estadísticas oficiales (Secretaría de Agricultura, Ganadería, Pesca y Alimentación 2006), es el tercer género en el país respecto a volúmenes extraídos anualmente, por debajo de los géneros Pinus y Eucalyptus. En la provincia de Buenos Aires se extrae el mayor volumen de productos forestales de Populus sp., con un total de 181.495 t/año (Secretaría de Agricultura, Ganadería, Pesca y Alimentación 2006). Posee esta provincia el 35\% de las forestaciones del género en el país, abasteciendo de materia prima a un mercado que consume anualmente unas 400.000 toneladas (Climent 1992, Dirección de Recursos Forestales Nativos 1995).

Las plantaciones de álamos situadas en los principales núcleos productivos del país son frecuentemente atacadas por Platypus mutatus Chapuis (=Platypus sulcatus, Coleoptera, Platypodidae), "taladrillo de los forestales". Adultos y larvas del insecto producen galerías en el fuste del hospedante, que pueden llegar a provocar la quebradura y muerte de la planta. Esta situación, entre otros efectos negativos, genera drásticas disminuciones de la producción por unidad de superficie en cantidad y calidad, dificulta las posibilidades de manejo de los rebrotes como continuidad de la explotación luego del corte (por la pérdida de pies correspondientes a ejemplares quebra- 
dos), y ocasiona inconvenientes en el aprovechamiento mediante cosecha mecánica, debido a los planos de debilidad generados en los fustes, aumentando significativamente los costos de explotación. Brugnoni (1980) y Toscani (1991), al referirse a la plaga en la región, le adjudican el rol de factor crítico para la producción. La European and Mediterranean Plant Protection Organization (EPPO) incluyó en 2004 a la plaga en el "listado de alerta" por su potencial peligrosidad.

Desde hace más de dos décadas, en la protección de los cultivos contra las causas adversas de origen animal y vegetal, ha tomado verdadera importancia la aplicación de estrategias de control integrado de plagas (INTA-FAO 1979). Su importancia ha ido aumentando dentro de la sanidad vegetal, perfilándose como la única salida razonable frente al desequilibrio causado por la exclusiva dependencia de los plaguicidas. Dentro de las tácticas de las que se vale, la resistencia varietal o resistencia genética de las plantas cultivadas con respecto a los artrópodos, resulta de gran importancia y se prevé que será cada vez más utilizada, debido a las grandes ventajas que presenta (Vigiani 1990).

La resistencia a los insectos es la propiedad hereditaria que le permite a la planta inhibir el crecimiento de las poblaciones de insectos, o recobrarse del daño causado por poblaciones que no fueron inhibidas. La inhibición del crecimiento de la población generalmente se deriva de las características morfológicas y bioquímicas de la planta, las cuales afectan el comportamiento o el metabolismo de los insectos hasta reducir el grado relativo de daño que podrían causar (Kogan 1992).

A nivel mundial, la selección de salicáceas ha sido tradicionalmente dirigida según criterios de vigor, rápido crecimiento, calidad de la madera y resistencia a enfermedades (Augustin et al. 1992), habiendo pocos estudios relacionados con resistencia-susceptibilidad a insectos; sin embargo, existen algunos emprendimientos que indican una posibilidad cierta de lograr buenos resultados desarrollando esa alternativa (Thielges, citado por Nef 1992). En Argentina, como parte de programas de mejoramiento genético de álamos, el Instituto Nacional de Tecnología Agropecuaria (INTA) trabaja en el establecimiento de un protocolo de cultivo de tejidos eficiente para clones de Populus deltoides, para ser utilizado con aquellos que aparezcan como poco susceptibles (Marinucci et al. 2004).

Dentro de la bibliografía referida a experiencias en Argentina, se pueden mencionar observaciones que indicarían que Populus deltoides cv. Harvard ( $P d$ I-63/51) y P. $x$ euroamericana cv. Conti 12 (Toscani 1990) y Populus deltoides cv. Catfish 2 (Casaubón 1993), serían clones de álamos comparativamente más susceptibles al ataque. Cerrillo (1996) menciona la existencia de estudios en ensayos comparativos de clones en los cuales no se presentaron diferencias significativas en cuanto al ataque.
Ante la situación expuesta, la posibilidad de entablar un plan de mejoramiento genético del álamo se presenta como alternativa poco explorada, pero viable, para desarrollar una técnica de control de Platypus mutatus. Para ello es imprescindible la realización de estudios que permitan caracterizar el efecto de la plaga sobre distintos clones, para identificar caracteres de resistencia al insecto en los resistentes o menos susceptibles. El objetivo del presente trabajo fue caracterizar la incidencia de Platypus mutatus en varios clones de Populus sp. de uso comercial, a través del número de galerías generado en el fuste, quiebres y supervivencia de ejemplares quebrados.

\section{MÉTODOS}

Los estudios se realizaron en plantaciones comerciales ubicadas en el Establecimiento Forestal "María Dolores", situado en el Partido de Alberti, provincia de Buenos Aires, República Argentina. Se trató de plantaciones con 10 años de edad, ubicadas todas dentro de un sector de unas 20 ha. La configuración de plantación correspondiente fue de $2,7 \mathrm{~m}$ entre filas, y una alternación constante de $3,5 \mathrm{~m}$ y $2,7 \mathrm{~m}$ entre plantas (densidad de 1.250 árboles por hectárea aproximadamente).

Los clones y cantidad de ejemplares disponibles para el estudio fueron los siguientes:

Populus deltoides cv. Catfish 2: 3.798 árboles, Populus deltoides cv. Stoneville 62: 2.675 árboles, Populus deltoides cv. Stoneville 66: 2.238 árboles, Populus deltoides cv. Stoneville 71: 5.502 árboles, Populus x euroamericana cv. 'I-214': 2.025 árboles y Populus. $x$ euroamericana cv. Conti 12: 3.638 árboles.

Selección de muestras. Mediante muestreo dirigido (en función del tamaño de plantas, no del ataque del insecto) se seleccionó una muestra de 54 árboles de cada clon, que cubrieran homogéneamente las tres clases diamétricas a estudiar, correspondientes a un rango de 15 a $30 \mathrm{~cm}$, con un intervalo de clase de $5 \mathrm{~cm}$ (18 ejemplares por clon, por clase diamétrica). Las clases inferiores a $15 \mathrm{~cm}$ se dejaron de lado, debido a que, según Núñez Cresto (1997), en general el ataque ocurre en ejemplares con un diámetro a la altura del pecho (dap) superior a $14 \mathrm{~cm}$; las clases mayores a $30 \mathrm{~cm}$ no se estudiaron, por resultar muy poco frecuentes en las plantaciones en estudio.

Recuento de galerías. Los árboles seleccionados fueron apeados y trozados cada $1 \mathrm{~m}$ para su inspección (en la primera troza se descontó la altura de la base o tocón). Dado que las cicatrices correspondientes a orificios de galerías ocluidas se confunden fácilmente con otras marcas de la corteza, para cerciorarse de su existencia, se realizó una muesca con hacha hasta poner en evidencia el orificio. Se registró el número de galerías presentes en cada troza del árbol completo. 
Representatividad de las muestras. Cada grupo de 18 ejemplares (iguales clon y clase diamétrica) fue dividido en forma aleatoria en tres submuestras de seis ejemplares. A cada submuestra se le estableció el valor de la mediana de los números totales de galerías por ejemplar y se calculó su media (M). Con los tres valores se cálculo el error estándar de la media de medianas correspondiente $\left(\mathrm{S}_{\mathrm{M}}\right)$. Con ambos valores y el valor de $\mathrm{t}$ de Student para n-1 grados de libertad, se calculó el intervalo de confianza para una probabilidad del $95 \%$ :

$$
\text { IC }(95 \%)=M \pm(“ t ”) S_{M}
$$

Análisis de los valores totales de galerías por ejemplar. Se efectuaron pruebas de Kruskal-Wallis agrupando los datos por clones, por clases diamétricas, y por clones y clases diamétricas. Se efectuaron pruebas de comparaciones múltiples usando el test de Nemenyi (Zar 1996).

Quebraduras ocasionadas por la plaga, según clones y supervivencia de ejemplares quebrados. En el total de ejemplares disponibles para el estudio se realizó un muestreo sistemático al 50\%, inventariando intercaladamente tres filas sí y tres filas no. Se tomaron como datos: a) árbol quebrado o entero y b) árbol quebrado vivo o árbol quebrado muerto. Los datos de árbol quebrado o entero se expresaron en porcentajes por unidad de registro (los grupos de tres filas) por clon. Para su tratamiento estadístico se transformaron los datos según la fórmula arcosen $\sqrt{ } \mathrm{Y}$, recomendada para datos binomiales expresados en porcentaje (Steel y Torrie 1997), siendo Y el valor porcentual expresado como fracción decimal. Con los datos transformados se realizó un análisis de la varianza y, al producirse diferencias significativas, se realizó un test de comparación de medias de Tukey. La rutina se realizó con Statgraphics Plus para Windows 4.0, precediendo a los análisis una comprobación de normalidad y homogeneidad de la varianza de los datos transformados efectuada por el mismo paquete estadístico (de ser necesario, se probarían otras transformaciones).

A los datos de árbol quebrado vivo y árbol quebrado muerto se les dio el mismo tratamiento expresado en el párrafo anterior (considerando como $100 \%$ al número de ejemplares quebrados por unidad de registro)

\section{RESULTADOS}

El número de galerías detectadas por ejemplar se incrementó al considerar clases diamétricas mayores (cuadro 1). Esto sucedió en términos generales y para cada clon en particular. El intervalo de confianza para cada mediana siempre se estableció a menos de dos unidades de la misma. Esta distancia resultó proporcionalmente menor a medida que aumentó el valor de la mediana, tanto en consideración a clones de un mismo tamaño como a distintos tamaños para un mismo clon.

La prueba de Kruskal-Wallis para los números de galerías por ejemplar, cuando se agruparon los datos por clon, generó un $\mathrm{H}$ con valor de 6,992 $(P=0,022) \mathrm{y}$, cuando se agruparon los datos por clase diamétrica, generó un $\mathrm{H}$ de $8,248(P=0,0162)$. Estos valores indicaron diferencias en las localizaciones de los grupos (cuadro 2).

$\mathrm{El}$ valor de $\mathrm{F}$ del análisis de la varianza correspondiente a los porcentajes transformados de árboles quebrados por clon resultó ser de 31,795 ( $P<0,0001)$. El quiebre de los ejemplares estuvo influenciado por el material genético involucrado: los porcentajes de ejemplares quebrados por clon fueron diferentes (cuadro 3).

El valor de $\mathrm{F}$ del análisis de la varianza correspondiente a los porcentajes transformados de árboles sobrevivientes por clon resultó ser de 33,856 ( $P<0,0001)$. El porcentaje de sobrevivencia de ejemplares estuvo influenciado por el material genético involucrado: los porcentajes de ejemplares quebrados por clon fueron diferentes (cuadro 4).

Cuadro 1. Mediana de los números de galerías por ejemplar, según clon y según clases diamétricas. Intervalos de confianza (IC) al $95 \%$.

Median of number of galleries by tree, according to clone and diameter at breast height classes. Confidence Intervals $(95 \%)$.

\begin{tabular}{|c|c|c|c|c|c|c|c|c|c|}
\hline \multirow[t]{2}{*}{ Clon } & \multicolumn{3}{|c|}{$\begin{array}{c}\text { Clase diamétrica }(\mathrm{cm}) \\
15-20\end{array}$} & \multicolumn{3}{|c|}{$\begin{array}{l}\text { Clase diamétrica }(\mathrm{cm}) \\
20-25\end{array}$} & \multicolumn{3}{|c|}{$\begin{array}{l}\text { Clase diamétrica }(\mathrm{cm}) \\
25-30\end{array}$} \\
\hline & Mediana & & $5 \%)$ & Mediana & $\mathrm{IC}$ & $5 \%)$ & Mediana & IC & $95 \%)$ \\
\hline Pxe Conti 12 & 11 & 9,8 & 12,2 & 30 & 28,7 & 31,3 & 44 & 42,1 & 45,9 \\
\hline Pd Stoneville 66 & 2 & 1,7 & 2,3 & 31 & 29,6 & 32,4 & 32 & 30,3 & 33,7 \\
\hline$P d$ Catfish 2 & 6 & 4,8 & 7,2 & 13 & 11,2 & 14,8 & 25 & 23,4 & 26,6 \\
\hline Pd Stoneville 62 & 3 & 1,9 & 4,1 & 7 & 6,0 & 8,0 & 16 & 14,0 & 18,0 \\
\hline Pd Stoneville 71 & 4 & 2,9 & 5,1 & 10 & 8,1 & 11,9 & 13 & 11,0 & 15,0 \\
\hline Pxe I-214 & 1 & 0,7 & 1,3 & 3 & 2,6 & 3,5 & 6 & 5,2 & 6,8 \\
\hline
\end{tabular}


Cuadro 2. Grupos de clones por clases diamétricas según la prueba de Nemenyi para el número de galerías por ejemplar.

Groups of clones by diameter at breast height classes according to Tukey test for mean number of galleries by tree.

\begin{tabular}{|c|c|c|c|}
\hline \multirow{2}{*}{ Clon } & \multicolumn{3}{|c|}{${ }^{1}$ Grupos homogéneos } \\
\hline & $15-20 \mathrm{~cm}$ & $20-25 \mathrm{~cm}$ & $25-30 \mathrm{~cm}$ \\
\hline Pxe I-214 & A & A & A \\
\hline Pd Stoneville 66 & A & $\mathrm{C}$ & C D \\
\hline$P d$ Stoneville 62 & A & $\mathrm{AB}$ & B \\
\hline$P d$ Stoneville 71 & A & $\mathrm{AB}$ & B \\
\hline$P d$ Catfish 2 & A B & B & $\mathrm{B} \mathrm{C}$ \\
\hline Pxe Conti 12 & B & $\mathrm{C}$ & $\mathrm{D}$ \\
\hline
\end{tabular}

1 Los tratamientos con letras iguales agrupan rangos sin diferencias significativas $(P=<0,05)$.

Cuadro 3. Grupos de clones por porcentaje de árboles quebrados, según la prueba de Tukey. of broken trees.

Groups of clones according to Tukey test for percentage

\begin{tabular}{lcc}
\hline \multicolumn{1}{c}{ Clon } & Media & $\begin{array}{c}{ }^{1} \text { Grupos } \\
\text { homogéneos }\end{array}$ \\
\hline Pxe I-214 & 0 & $\mathrm{~A}$ \\
Pd Stoneville 62 & 0,5 & $\mathrm{~B}$ \\
Pd Stoneville 71 & 0,6 & $\mathrm{~B}$ \\
Pxe Conti 12 & 2,4 & $\mathrm{C}$ \\
Pd Stoneville 66 & 5,0 & $\mathrm{CD}$ \\
Pd Catfish 2 & 7,3 & $\mathrm{D}$ \\
\hline
\end{tabular}

1 Los tratamientos con letras iguales agrupan rangos sin diferencias significativas $(P=<0,05)$.

Cuadro 4. Grupos de clones por porcentaje de árboles quebrados sobrevivientes, según la prueba de Tukey.

Groups of clones according to Tukey test for percentage of surviving broken trees.

\begin{tabular}{lrc}
\hline \multicolumn{1}{c}{ Clon } & Media & $\begin{array}{c}\text { 'Grupos } \\
\text { homogéneos }\end{array}$ \\
\hline$P d$ Stoneville 71 & 100 & $\mathrm{~A}$ \\
$P d$ Stoneville 66 & 96 & $\mathrm{~A}$ \\
$P d$ Stoneville 62 & 89 & $\mathrm{~B}$ \\
Pd Catfish 2 & 84 & $\mathrm{~B}$ \\
Pxe Conti 12 & 9 & $\mathrm{C}$ \\
\hline
\end{tabular}

1 Los tratamientos con letras iguales agrupan rangos sin diferencias significativas $(P<0,05)$.

\section{DISCUSIÓN}

La preferencia del insecto por los mayores diámetros (cuadros 1 y 2) concuerda con lo expresado, entre otros, por Santoro (1957, 1963), Bascialli et al. (1993), Cerrillo (1996) y Núñez Cresto et al. (2001). Núñez Cresto et al. (2001) realizaron sus estudios en un clon de la especie que participó de este ensayo: Populus deltoides Catfish 2 ; estudiaron el comportamiento del par insecto-hospedante en dos sitios diferentes, siendo los resultados concordantes en cuanto a la preferencia por los mayores tamaños. Santoro (1963) expresó que el insecto, por algún mecanismo biológico, selecciona los diámetros mayores para disponer de espacio suficiente para poder criar una gran cantidad de larvas.

La influencia que ejerce el material genético (cuadro 2) concuerda con lo expresado por Toscani (1990) y Casaubón et al. (1993), que aportan información que respaldaría la hipótesis de un diferencial de susceptibilidad clonal. En contraposición, Cerrillo (1996) no detectó diferencias para un grupo de clones del género en estudio. Esta situación presentada para las tres clases diamétricas analizadas (cuadros 1 y 2 ) implica diferentes comportamientos de los clones ya a partir de los menores tamaños susceptibles de ser atacados.

Los clones $P x e$ I-214, $P d$ Stoneville 71 y $P d$ Stoneville 62 se presentan como los menos afectados por perforaciones de galerías (cuadros 1 y 2). Pxe Conti 12 aparece como el clon más expuesto a ser perforado por el accionar del insecto para las tres clases diamétricas (cuadros 1 y 2). Toscani (1990) califica a este clon como susceptible a la plaga, aunque sin mencionar la metodología de comparación utilizada que lo indujo a expresar el comentario. $P d$ Stoneville 66 está en el grupo de los más afectados al tratarse de las clases diamétricas superiores (cuadros 1 y 2), donde se maximizan los daños económicos en la calidad del producto a cosechar. $P d$ Catfish 2, que integra un segundo grupo por presencia de galerías (cuadros 1 y 2), fue caracterizado por Casaubón et al. (1993) como un material genético muy susceptible al ataque, en un estudio que, si bien no focalizaba en el estudio de la plaga, aporta observaciones en distintos aspectos del comportamiento de un grupo de nueve clones del género Populus; los resultados específicos para este clon resultan de particular importancia por ser uno de los más difundidos en los sectores productivos del país (Petray 1998ab).

Existen coincidencias y diferencias entre el estudio de número de galerías por clon (cuadros 1 y 2) y el de porcentaje de árboles quebrados por clon (cuadro 3). $P d$ Catfish 2, señalado por Casaubón et al. (1993) como susceptible al ataque, se ubica en el grupo de los clones que presentan mayor porcentajes de quiebre; sin embargo, no se instala en el grupo de los portadores de mayor número de galerías. $P d$ Stoneville 66 se integra a los grupos más afectados tanto por presencia de galerías 
como quiebres detectados. $P$ x e Conti 12 se sitúa entre los más afectados por presencia de galerías, pero no entre los que cuentan con mayor porcentaje de ejemplares quebrados.

No se establece, entonces, una asociación estrecha entre número de galerías y condición de quebradizo. La misma está condicionada por el clon. Esta discordancia podría estar ligada a características mecánicas del leño, como densidad, rigidez y dureza. Estas características son consecuencia de diferentes factores, como son la cantidad de leño de verano, el espesor de las paredes de las fibras, el tamaño de las células y su proporción relativa, y la composición química de la madera (Bonavía de Guth 1981, 1982, 1987, Bonavía de Guth y Piussan 1989). La densidad de la madera puede variar entre especies, entre árboles de una misma especie y dentro del árbol. Para distintos clones de álamos han sido detectadas diferencias (Bonavía de Guth 1982, 1989, Yanchuck et al. 1983), y esta situación podría condicionar el efecto de la plaga en los clones

La condición de un clon de ser quebradizo por influencia de la plaga, por un lado, disminuye la calidad y valor comercial de la cosecha por impedir la utilización del $100 \%$ del fuste y, por otro, restringe las alternativas mecanizadas de cosecha, por la posibilidad de quiebre durante la misma.

El clon Pxe Conti 12 (cuadro 4) es el que presenta la menor supervivencia de árboles quebrados, condición que podría estar relacionada con una menor tolerancia a la sombra. El hecho de no sobrevivir los ejemplares quebrados reduce el número de cepas efectivas remanentes, circunstancia de peso al momento de decidir si se maneja el rebrote o se replanta el cuadro.

$P d$ Stoneville 71 que ocupa una posición intermedia entre los afectados por quiebre y presencia de galerías, no presenta mortandad en los ejemplares quebrados.

La plaga, por cantidad de galerías generadas, porcentaje de árboles quebrados producidos y muertes inducidas, impacta de distinta manera a los clones estudiados. Para cada uno de los efectos estudiados hay distintas respuestas, según el clon; para cada clon, el nivel de impacto de la plaga puede variar, según la afectación analizada (galerías, quiebre, muerte). Esto implica, entre otros, la necesidad de contar con información en particular por material genético, acerca de los distintos efectos de la plaga en las plantaciones, al momento de decidir clones a utilizar, modalidades de cosecha y manejo de rebrotes o reemplazo de cepas.

Se cree conveniente replicar este tipo de experiencias en otros ambientes, variando suelos y condiciones climáticas, para que al cambiar las condiciones en que se desarrolla la plaga y se genera el leño, observar el comportamiento de las asociaciones establecidas.

\section{CONCLUSIONES}

Platypus mutatus produce distintos efectos en diferentes clones del Género Populus.

El ordenamiento comparativo entre clones, según nivel de afectación recibido, resulta variable de acuerdo a la perturbación analizada (número de galerías presentes, quiebre del fuste, muerte del ejemplar quebrado).

Dentro del presente estudio, Pxe Conti 12 y $P d$ Stoneville 66 aparecen como los clones más afectados por la presencia de galerías. Los clones Pxe I-214, Pd Stoneville 71 y $P d$ Stoneville 62 se presentan como los menos afectados por perforaciones de galerías.

$P d$ Catfish 2 y $P d$ Stoneville 66, seguidos por el clon $P x e$ Conti, se presentan como más propensos al quiebre. El clon Pxe I-214 no se ve afectado por quebraduras.

Los ejemplares quebrados de Pxe Conti 12 tienen pocas chances de supervivencia. Los de $P d$ Stoneville 71 no mueren, a pesar de las quebraduras. Los de $P d$ Stoneville 62, $P d$ Stoneville 66, y $P d$ Catfish 2, presentan alta supervivencia de ejemplares quebrados.

Estas asociaciones establecidas entre clones y efectos de la plaga deben ser puestas a prueba en otros ambientes.

\section{REFERENCIAS}

Augustin S, J Chenault, D Cornu, A Delplanque, L Jouanin, JC Leple, S Picard, M Villar. 1992. Informe sobre las investigaciones del INRA (Francia) acerca de la resistencia de los álamos contra los insectos. $19^{\mathrm{a}}$ Sesión de la Comisión Internacional del Álamo. Zaragoza, España, vol. 1, p. 355360.

Bascialliascialli M, AE Etienot, RA Giménez, H Toscani, MC Tuozzo. 1993. Análisis de control químico de Platypus sulcatus (Chapuis) en plantaciones comerciales de álamo. Congreso Forestal Argentino y Latinoamericano. Paraná, Argentina. p. 41-50.

Bonavia de Guth E, CM Piussan. 1989. Variación de las características del leño del individuo en Salix nigra cultivado en el Delta del Paraná. Centro de Investigaciones y Experiencias Forestales, Simposio sobre Silvicultura y Mejoramiento Genético de Especies Forestales. Buenos Aires, Argentina. p. 219-235.

Bonavia de Guth E. 1981. Características del leño de varias especies de Salix en apoyo a la obtención de buenos clones papeleros. Congreso de ATIPCA. Buenos Aires, Argentina. p. 25-32.

Bonavia de Guth E. 1982. Evaluación de varios híbridos obtenidos por cruzamiento de Salix alba x S. matsudana. Congreso de ATIPCA. Buenos Aires, Argentina. p. 19-30.

Bonavia de Guth E. 1987. Obtención y evaluación de nuevos clones de sauces y álamos en relación a su uso celulósico. Congreso de ATIPCA. Buenos Aires, Argentina. p. 4360.

Brugnoni HC. 1980. Plagas Forestales. Buenos Aires, Argentina. Hemisferio Sur. p. 152-157. 
Casaubón EA, LB Gurini, SC Cortizo. 1993. Evaluación dasométrica de nueve clones de $P$. deltoides cultivados en el Delta del Río Paraná. Congreso Forestal Argentino y Latinoamericano. Paraná, Argentina. p. 57-62.

Cerrillo T. 1996. Revisión bibliográfica sobre Platypus sulcatus Chapuis y otros coleópteros del género. Revista de la Asociación Forestal Argentina 1(5):59-70.

Climent M. 1992.Consideraciones sobre el uso del álamo para celulosa. Seminario sobre costos y rentabilidad forestal. Asociación Forestal Argentina. Buenos Aires, Argentina. p. 54-67.

Dirección Recursos Forestales Nativos. 1995. Anuario de Estadísticas Forestales. Buenos Aires, Argentina. 7 p.

INTA-FAO. 1979. Compendio del Curso de Perfeccionamiento en Control Integrado de Plagas. Pergamino, Argentina. EERA-INTA. $135 \mathrm{p}$.

Kogan M. 1992. Resistencia de la planta en el manejo de Plagas. In Metcalf RL, WH Luckmann eds. Introducción al Manejo de Plagas de Insectos. México D.F., México. LIMUSA. vol. 4, p. 123-172.

Marinucci L, S Sharry, W Abedini. 2004. Transformación genética del álamo para resistencia a Platypus sulcatus. Establecimiento de un protocolo de cultivo de tejidos eficiente para los clones de Populus deltoides cv. "Catfish 2" y"Catfish 5". SAGPyA Forestal 32:42.

Nef L, F Duhoux. 1992. Poplar and Willow resistance to insects. $19^{a}$ Sesión de la Comisión Internacional del Álamo. Zaragoza, España, vol. 1. p. 307-325.

Núñez Cresto M, JL Marquina, RM Marlats. 2001. Taladrillo de los forestales (Platypus sulcatus Chapuis): en Populus deltoides Marsh. cv. Catfish 2. Revista de la Facultad de Ciencias Agrarias (Argentina) 33 (2):31-40.

Núñez Cresto MC. 1997. Informe de Beca de Estudio: Estudio de la susceptibilidad clonal del álamo (Populus sp.) al ataque de Platypus sulcatus Chapuis (Taladrillo de los forestales). Comisión de Investigaciones Científicas de la Provincia de Buenos Aires. Argentina. 50 p.

Petray E. 1998a. Evolución de las Forestaciones realizadas con Salicáceas en la Provincia de Buenos Aires en el Contexto del Régimen de Promoción de Plantaciones Forestales. Secretaría de Agricultura, Ganadería, Pesca y Alimenta- ción, 1998, Informe interno de la Dirección de Producción Forestal. Buenos Aires, Argentina. p. 123-124.

Petray E. 1998b. Evolución de las forestaciones realizadas con Salicáceas (Populus y Salix) en la Provincia de Entre Ríos (1992-1995) en el Contexto del Régimen de Promoción de Plantaciones Forestales. Secretaría de Agricultura, Ganadería, Pesca y Alimentación. Informe interno de la Dirección de Producción Forestal. Buenos Aires, Argentina. p. 14-15.

Santoro H. 1957. Contribución al conocimiento de la biología de Platypus sulcatus Chapuis (taladro del "álamo Mussolini" y de otras especies forestales) (Coleoptera, Platipodidae). Revista de Investigaciones Forestales (Argentina) 3(1):7-19.

Santoro H. 1963. Bioecología de Platypus sulcatus Chapuis (Coleoptera, Platipodidae). Revista de Investigaciones Forestales (Argentina) 4 (1):47-79.

Santoro H. 1963. Nuevo antecedente de la lucha manual contra Platypus sulcatus Chap. (Coleoptera, Platypodidae) IDIA Supl. Forestal $\mathrm{N}^{\circ}$ 4:70-74.

Secretaría de Agricultura, Ganadería, Pesca y Alimentación. 2002. Guía Forestal. Buenos Aires, Argentina Secretaría de Agricultura, Ganadería, Pesca y Alimentación, 2a edición. p. 107-108.

Steel RGD, JH Torrie. 1997. Bioestadística. Principios y procedimientos. México D.F., México. McGraw-Hill. p. 226-230.

Toscani HA. 1990. Manual para la protección de los cultivos forestales en la región del Delta del Paraná. 35 Reunión Comisión Internacional del Alamo. Informe Comité Ejecutivo. Buenos Aires, Argentina. 17 p.

Toscani HA. 1991. Manual para la protección de los cultivos forestales de la Región del Delta del Río Paraná. Buenos Aires, Argentina. Comisión Internacional del Álamo-FAO. Secc. 13. p. 1-11.

Vigiani RA. 1990. Hacia el control integrado de Plagas. Buenos Aires, Argentina. Hemisferio Sur. 122 p.

Yanchuck AD, BP Dancik, MM Micko. 1983. Intraclonal variation in wood density of Trembling Aspen in Alberta. Wood and Fiber Science 15 (4):387-394.

Zar JH. 1996. Biostatistical Analysis. USA. Electronic Technical Publishing. p. 226-227. 